\title{
Nanoindentation of thin graphdiyne films: Experiments and molecular dynamics simulation
}

\author{
Kailu Xiao a, c, Jiaofu Li ${ }^{\text {b, c }}$, Xianqian $\mathrm{Wu}^{\text {a, c, *, Huibiao Liu }}{ }^{\text {b, c, ** }}$, Chenguang Huang ${ }^{\text {a, c }}$, \\ Yuliang $\mathrm{Li}^{\mathrm{b}, \mathrm{c}}$ \\ ${ }^{a}$ Key Laboratory of Mechanics in Fluid Solid Coupling Systems, Institute of Mechanics, Chinese Academy of Sciences, Beijing, 100190, China \\ b Beijing National Laboratory for Molecular Sciences (BNLMS), CAS Key Laboratory of Organic Solids, Institute of Chemistry, Chinese Academy of Sciences, \\ Beijing, 100081, China \\ ${ }^{\mathrm{c}}$ School of Engineering Science, University of Chinese Academy of Sciences, Beijing, 100049, China
}

\section{A R T I C L E I N F O}

\section{Article history:}

Received 9 July 2018

Received in revised form

30 November 2018

Accepted 9 December 2018

Available online 10 December 2018

\begin{abstract}
A B S T R A C T
Graphdiyne possesses not only high strength but also excellent ductility, making it possible to be used in future high-performance protective structures. In this paper, the mechanical properties of graphdiyne were firstly measured by AFM experiments, and the failure behavior during low velocity perforation was also investigated by molecular dynamics (MD) simulations. Firstly, the elastic modulus was measured to be about 218.5 GPa by AFM experiments, which is about half of its ideal value due to various defects and the layer numbers of the synthesized graphdiyne film. Then, the nanoindentation processes of graphdiyne films were investigated by MD simulations, and the elastic modulus and strength were simulated to be about $489.04 \mathrm{GPa}$ and $33.95 \mathrm{GPa}$, respectively. The failure behavior of the graphdiyne film was also studied in atomic level. Sequential broken of $C \equiv C, C=C$ and $C-C$ bonds and recombination of the broken bonds were observed to form a unique lathy crack. Furthermore, the effects of loading speed and indenter radius on the mechanical response of graphdiyne were investigated. A revised formula was developed for analyzing the mechanical properties of films in AFM experiments under various loading conditions.
\end{abstract}

() 2018 Elsevier Ltd. All rights reserved.

\section{Introduction}

Two-dimensional (2D) materials have attracted great attention due to excellent properties such as high strength and high thermal conductivity after graphene is discovered. It has found various potential applications such as electricity, chemistry, and energy storage [1-5]. Most of 2D materials have remarkable mechanical strengths [6-9] and high electronic performance [10,11]. Graphdiyne [12,13], one of the new carbon allotropes, exhibits outstanding properties, which derives from its unique structure consisting of $\mathrm{sp}^{2}$ and $\mathrm{sp}$ hybridized carbon atoms. The presence of acetylene can decrease the binding energy so that the electronic

\footnotetext{
* Corresponding author. Key Laboratory of Mechanics in Fluid Solid Coupling Systems, Institute of Mechanics, Chinese Academy of Sciences, Beijing, 100190, China.

** Corresponding author. Beijing National Laboratory for Molecular Sciences (BNLMS), CAS Key Laboratory of Organic Solids, Institute of Chemistry, Chinese Academy of Sciences, Beijing, 100081, China.

E-mail addresses: wuxianqian@imech.ac.cn (X. Wu), liuhb@iccas.ac.cn (H. Liu).
}

and optical properties can be modulated, which is superior to graphene and carbon nanotubes [14,15].

Unlike graphene, the structure of graphdiyne has diacetylene $(-\mathrm{C} \equiv \mathrm{C}-\mathrm{C} \equiv \mathrm{C}-$ ) linkages between benzene rings, making it more flexible and ductile than graphene. Nevertheless the strength of the graphdiyne will be sure to lower because of lower atom density when compared to graphene. It is well known that the strength and ductility of a material has always been competitive mechanism. However, graphdiyne could balance the competition between its strength and ductility in some extent, indicating its promising application in engineering industries such as impact protective coating for micro-projectiles. There is considerable body of literature that addresses the mechanical behavior of graphdiyne by theoretical analysis and MD simulation. Cranford et al. [16] derived basic scaling laws for the cumulative effects of additional acetylene repeats through a spring-network framework, allowing prediction of mechanical performance of other extended graphdiyne structures. Based on their calculation, the in-plane stiffness of a monolayer graphdiyne was estimated to be $170.4 \mathrm{~N} / \mathrm{m}$, and the strength 
was estimated to be $45 \mathrm{GPa}$ in zigzag direction and $29 \mathrm{GPa}$ in armchair direction. Sergio et al. [17] calculated the Young's modulus, Poisson's ratio, and linear thermal expansion coefficient of seven types of graphynes and graphdiynes using classical MD simulation. The ab initio simulation by Ahangari et al. [18] indicated that the in-plane stiffness and Young's modulus of graphdiyne decreased from $164.4 \mathrm{~N} / \mathrm{m}$ and $513.8 \mathrm{GPa}$ at room temperature to $128.9 \mathrm{~N} / \mathrm{m}$ and $402.8 \mathrm{GPa}$ at $1500 \mathrm{~K}$, respectively. It has been observed that in the same range of strain, larger force could be exerted to graphene than to graphdiyne. Pei [19] investigated the influences of strain on the energetic and electronic properties of graphdiyne based on first-principles calculations. The in-plane stiffness and Poisson's ratio of graphdiyne were calculated to be $7.60 \mathrm{eV} / \AA^{2}$ and 0.453 , respectively. Yang et al. [20] calculated the mechanical properties of monolayer graphdiyne under tensile loading by MD simulations. The fracture strains and associated ultimate stresses were also obtained.

Graphdiyne was firstly synthesized on the surface of copper in 2010 by Li et al. [13] through a cross-coupling reaction using hexaethynylbenzene. After that it has been extensively studied in various application conditions, such as field emission [21,22], energy storage [23,24], catalysis [25,26] and energy conversion $[27,28]$. To the best of our knowledge, the mechanical behavior of graphdiyne has not been investigated by experiments previously. Especially, the strength and failure behavior of graphdiyne, which is important to estimate its responses in some challenging engineering such as micro-projectile impact protection, is not well understood.

In this paper, the elastic properties and failure behavior of graphdiyne was studied by experiments and MD simulation. Firstly, the indentation response of the graphdiyne film, which had been transferred to a copper network with square wells, was investigated by atomic force microscope (AFM). The Van der Waals interaction between the copper network and the graphdiyne films rendered strong binding condition. The experimental results showed that the graphdiyne film had excellent mechanical performance such as high strength, more flexible and stretchable when compared to graphene. Then, the failure behavior of the graphdiyne films was investigated by MD simulation. The sequential failure of the graphdiyne's bonds as well as the fracture initiation behavior of the graphdiyne was obtained, showing high ductility of the graphdiyne film. The effects of loading parameters such as diameter of the indenter and its moving velocity on the mechanical response of the graphdiyne film were also investigated by MD simulation.

\section{Experimental and MD simulation methods}

\subsection{Materials}

The graphdiyne films were synthesized according to Ref. [13] The copper foils were pretreated by sonicating in $1 \mathrm{M} \mathrm{HCl}$, acetone and ethanol subsequently for $15 \mathrm{~min}$, dried under a flow of argon, then used immediately for growing graphdiyne films. The monomer of hexaethynylbenzene was synthesized in good yield (62\%) by adding tetrabutylammonium fluoride (TBAF) to the THF solution of hexakis[(trimethylsilyl)ethynyl]benzene for $10 \mathrm{~min}$ at $8{ }^{\circ} \mathrm{C}$. The graphdiyne was grown on the surface of copper foil in the pyridine by a cross-coupling reaction of the monomer structure of hexaethynylbenzene for $72 \mathrm{~h}$ at $60^{\circ} \mathrm{C}$ under nitrogen atmosphere. In the procedure of forming graphdiyne, the copper foil was not only the catalyst for the cross-coupling reaction but also the substrate for growing graphdiyne films.

Then the successfully synthesized graphdiyne films were transferred to a copper network with distributed square wells with a dimension of $6.1 \times 6.1 \mu \mathrm{m}$ for preparing AFM experiments. The width between two adjacent wells was $5.1 \mu \mathrm{m}$. The Van der Waals interaction between the copper network and the transformed graphdiyne film made the graphdiyne film binding strongly with the boundary of the wells of the copper substrate.

\subsection{AFM experiments and analysis methods}

The thermo-mechanical noise method was employed to calibrate the spring constant by recording the thermal noise spectrum when the environmental temperature was balanced with the surrounding temperature of cantilever. According to the equipartition theorem and vibration amplitude when the cantilever is in firstorder inherent frequency, the spring constant can be calculated as follows,

$K=\frac{k_{B} T}{\left\langle Z_{c}^{2}\right\rangle}$

where $k_{B}$ is Boltzmann constant, $\left\langle Z_{c}^{2}\right\rangle$ represents mean square of harmonic oscillator displacement. The spring constant was determined as $25.47 \mathrm{~N} / \mathrm{m}$. Additionally, the optical leverage factor was $81.65 \mathrm{~nm} / \mathrm{V}$ by measuring the sensitivity of optical leverage system. A diamond tip was chosen to obtain the elastic behavior of the graphdiyne film in AFM experiments. The length of the indenter was $6.4 \pm 1 \mu \mathrm{m}$ and the radius of curvature of the indenter tip was $20 \mathrm{~nm}$ as shown in Fig. S1. Prior to indentation, the ac mode of the AFM was used to image the suspended graphdiyne films because ac mode was less likely to cause damage in specimen than contact mode. The graphdiyne films were scanned in the noncontact mode and a slightly withdraw was executed. Then the AFM tip was positioned within $60 \mathrm{~nm}$ of the center of the rectangular graphdiyne film to minimize the location error of the AFM tip. Finally, the mechanical tests were performed in the contact mode, and each graphdiyne film experienced a loading and unloading cycle.

In AFM experiments, the relationship between the loading force, $F$, and its corresponding deflection, $\delta$, could be described by a polynomial function according to the analytical model of a clamped circular elastic film under point loading $[29,30]$. The loading force, $F$, could be divided into two aspects,

$F=F_{\sigma^{2 D}}(\delta)+F_{E^{2 D}}(\delta)$,

where $\delta$ denotes deflection of film. The first item. $F_{\sigma^{2 D}}(\delta)$, represents the initial pre-tension of film, having a linear relationship between force and displacement. The second term, $F_{E^{2 D}}(\delta)$, is valid for applied stress larger than initial axial pre-tension stress, having a cubic relationship between force and displacement.

According to the loading theory of elastic film, there are different loading models according to the sizes of indenter and circular film. If $r / a>0.1$, where $r$ and $a$ are radii of indenter and film, respectively, the sphere load model is dominant, and the effect of the parameter $\mathrm{r} / \mathrm{a}$ should be taken into account. The relationship between the initial-tension force, $F_{\sigma^{2 D}}(\delta)$, and deflection of a clamped elastic film, $\delta$, under sphere load model has been derived by Wan et al. [29] and could be expressed as follows while neglecting the bending stiffness of the film,

$F_{\sigma^{2 D}}(\delta)=\sigma_{0}^{2 D}(\pi a)\left(\frac{r}{a}\right)^{3 / 4}\left(\frac{\delta}{a}\right)$

where $\sigma_{0}^{2 D}$ denotes pre-tension stress of film.

Based on the present AFM, a rectangle film is employed. Therefore, Eq. (2-3) could be revised by introducing the aspect 
ratio, $\beta$, as follows according to the study by Xiang et al. [31],

$F_{\sigma^{2 D}}(\delta)=\sigma_{0}^{2 D}(\pi a) \beta^{1 / 2}\left(\frac{r}{a}\right)^{3 / 4}\left(\frac{\delta}{a}\right)$

where $\sigma_{0}^{2 D}$ denotes pre-tension stress of film, $\beta$ denotes aspect ratio of sample, and $a=(l w / \pi)^{1 / 2}$ represents equivalent radius of rectangular graphdiyne film, where $l$ and $w$ are length and width, respectively.

For the applied force, $F_{E^{2 D}}(\delta)$, its relationship with the deflection, $\delta$, of a circular film has been given by Komaragiri et al. [30] In the present study, the relationship is also revised so that it is validate for a film with rectangle shape according to the study by Wang et al. [32],

$F_{E^{2 D}}(\delta)=E^{2 D}\left(q^{3} a\right) \beta^{1 / 2}\left(\frac{r}{a}\right)^{1 / 4}\left(\frac{\delta}{a}\right)^{3}$,

where $E^{2 D}$ denotes Young's modulus of 2D material, $q=1 /(1.049-$ $\left.0.146 v-0.158 v^{2}\right)=1.036$ is dimensionless constant, and $v=0.4$ is Poisson's ratio [17].

If $r / a<0.1$, the point loading model is dominant, and the effect of $r / a$ is negligible. The pre-tension term and the stress term can be expressed as follows,

$F_{\sigma^{2 D}}(\delta)=\sigma_{0}^{2 D}(\pi a) \beta^{1 / 2}\left(\frac{\delta}{a}\right)$,

$F_{E^{2 D}}(\delta)=E^{2 D}\left(q^{3} a\right) \beta^{1 / 2}\left(\frac{\delta}{a}\right)^{3}$

In the present study, the length and the width of the films are $6.10 \mu \mathrm{m}$. The equivalent radii of indenter and rectangle specimens are $20 \mathrm{~nm}$ and $3.44 \mu \mathrm{m}$ for AFM experiments and $20 \AA$ and $304.27 \AA$ for MD simulation. As a result, the point loading model is dominant in both experimental and MD simulation.

According to the study by Bhatia et al. [33], the maximum stress for an elastic rectangular film can be expressed as

$\sigma_{m}^{2 D}=\left(\frac{F_{\max } E^{2 D}}{8 \pi r}\right)^{1 / 2}$,

where $\sigma_{m}^{2 D}$ is maximum stress of film under indenter, $r$ is radius of indenter tip and $F_{\max }$ denotes breaking force under indentation.

\subsection{MD model}

The MD simulations through large scale atom/molecular massively parallel simulation (LAMMPS) package [34] were performed to simulate the mechanical behavior especially the failure behavior of graphdiyne during nanoindentation. The monolayer graphdiyne film was constructed to achieve high computational efficiency. The carbon atom number of the model was 81468 . There were three kinds of bonds between the adjacent carbon atoms, i.e. aromatic (e.g. $\mathrm{sp}^{2}$ ), single, and triple (e.g. $\mathrm{sp}^{1}$ ). The bond lengths were set to be $1.44 \AA$ for aromatic bonds, $1.40 \AA$ for single bonds, and $1.24 \AA$ for triple bonds, respectively [35]. Fig. 1 shows the MD model of the nanoindentation tests, where the upper sphere configuration represents the tip of the indenter as used in AFM experiments. Since the indenter was constructed by diamond, it was regarded as a rigid object in MD simulations to pursue high efficiency. The length and width of the graphdiyne film were $572 \AA$ and $578 \AA$, respectively, and the radius of the indenter was $20 \AA$. The point-loading model was taken to describe the interaction between the indenter and the film according to the theory of elastic.

Fixed boundary conditions were applied on all the sides of the graphdiyne film during simulation. The carbon-carbon interaction of the graphdiyne film was described by the adaptive intermolecular reactive empirical bond order (AIREBO) potential [36], which is a variant of the reactive empirical bond-order (REBO) potential. Both REBO and AIREBO are the membership of classical bond-order family of Tersoff-Brenner potentials, which could effectively describe the covalent bonds in non-polar systems. Compared with REBO, AIREBO potential not only includes multi-body potential effects, but also considers the long-range interactions and torsion term. It has been proved to be more accurate in describing bond breaking and reforming of carbon atoms than Tersoff potential [37]. It is worth to note that there should be a cutoff value of the interaction between the nearest neighbor atoms to accurately capture the bond breakage and re-hybridization. In the present study the cutoff value of AIREBO potential was set to be $2.0 \AA$ [38]. The interaction between the indenter and the graphdiyne film was described by Lennard-Jones (LJ) potential with $\sigma_{\mathrm{C}}=3.4 \AA$ and $\varepsilon_{\mathrm{C}}=2.84 \mathrm{meV}$, where $\sigma_{\mathrm{C}}$ is distance when potential is zero and $\varepsilon_{\mathrm{C}}$ is depth of potential wall in LJ potential. The NVT ensemble was taken during simulation. The temperature was controlled by NoseHoover algorithm, and the room temperature of $300 \mathrm{~K}$ was set to be equilibrium temperature. The velocity-verlet time stepping method was adopted, and the time-step was set to be 1 fs to achieve more accurate simulation results. Before the process of nanoindentation, the energy minimization and relaxation were carried out to ensure that the system stayed in the equilibrium state with lowest energy.

\section{Results and discussion}

\subsection{The characterization of graphdiyne films}

Fig. 2 shows the structure characterization of the graphdiyne films. Fig. 2a exhibits the morphology of synthesized graphdiyne films by scanning electron microscopy (SEM). The microstructure of the graphdiyne film has the similar morphology as 3D porous materials. Interestingly, the erected nanowalls exists submicrometer voids, and the diameters of those voids vary from tens of micrometer to hundreds of micrometer. The distribution of the nanowalls is significantly uniform and the synthesized film is thin as shown by the nanowalls. Fig. $2 \mathrm{~b}$ shows the transmission electron microscopy (TEM) observation of the graphdiyne film with a smooth surface. Fig. 2c shows the high resolution TEM image, which clearly exhibits interlayer space of $3.65 \AA$. All the results are consistent with the observation of Refs. [13,39-41]. Fig. 2d shows typical Raman spectra of the graphdiyne film in various randomly selected positions to distinguish the quality of the graphdiyne film before being transferred to the surface of copper foil. The Raman spectra in different positions show the same four prominent peaks at $1386.7 \mathrm{~cm}^{-1}, 1572.4 \mathrm{~cm}^{-1}, 1926.4 \mathrm{~cm}^{-1}$ and $2167.9 \mathrm{~cm}^{-1}$, respectively. The peak of the first-order scattering is $1572.4 \mathrm{~cm}^{-1}$, showing that the $\mathrm{sp}^{2}$ carbon domains in aromatic rings by $\mathrm{E}_{2 \mathrm{~g}}$ mode in-phase stretching vibration. The peak at $1386.7 \mathrm{~cm}^{-1}$ is attributed to the breathing vibration of $\mathrm{sp}^{2}$ carbon domains in aromatic rings. The peaks at $1926.4 \mathrm{~cm}^{-1}$ and $2167.9 \mathrm{~cm}^{-1}$ can be attributed to the vibration of conjugated diyne links $(-\mathrm{C} \equiv \mathrm{C}-\mathrm{C} \equiv \mathrm{C}-$ ). The intensity ratio of the peak at $1386.7 \mathrm{~cm}^{-1}$ and $1572.4 \mathrm{~cm}^{-1}$ is 0.788 , indicating the high order and low defects in the graphdiyne films. Fig. 2e shows the high resolution results of asymmetric $C 1 \mathrm{~s}$ of the graphdiyne film by X-ray photoelectron spectroscopy (XPS). The C $1 \mathrm{~s}$ peak can be de-convoluted into four sub-peaks at $288.23 \mathrm{eV}$, $286.44 \mathrm{eV}, 284.56 \mathrm{eV}$ and $285.03 \mathrm{eV}$, which has been specified as $\mathrm{C}$ $1 \mathrm{~s}$ orbital of $\mathrm{C}=\mathrm{O}, \mathrm{C}-\mathrm{O}, \mathrm{C}-\mathrm{C}(\mathrm{sp})$ and $\mathrm{C}-\mathrm{C}\left(\mathrm{sp}^{2}\right)$, respectively, after 


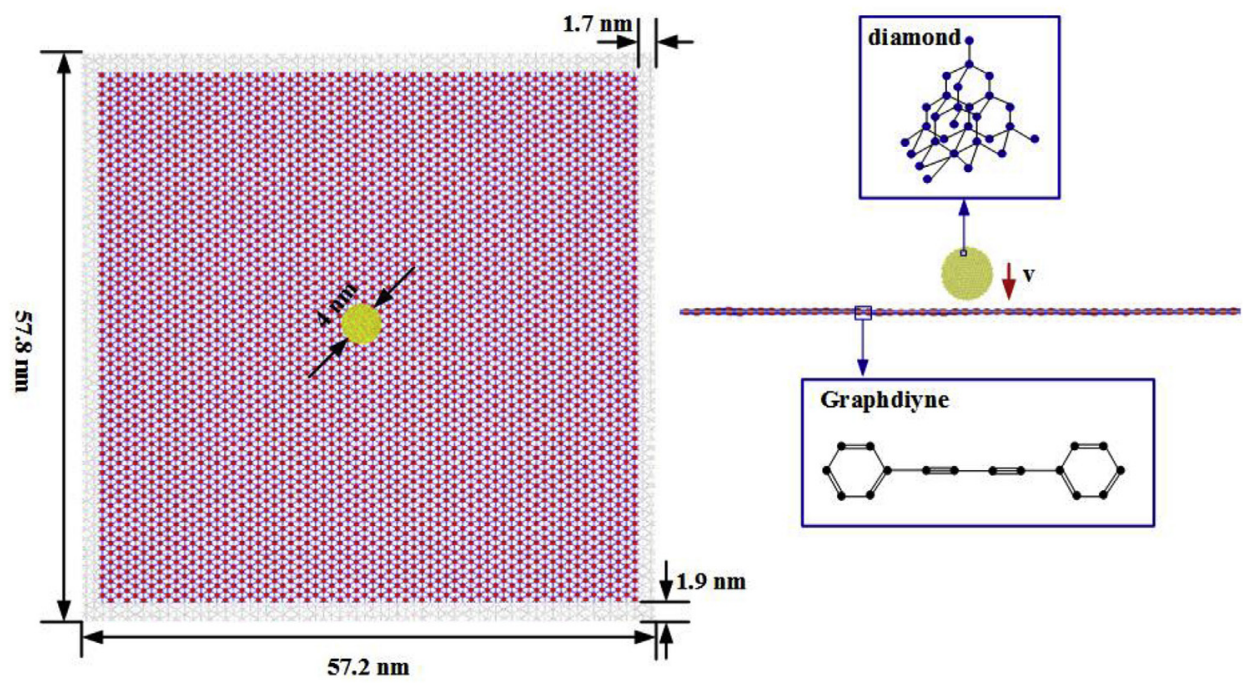

Fig. 1. Atomic configuration of MD model. (A colour version of this figure can be viewed online.)
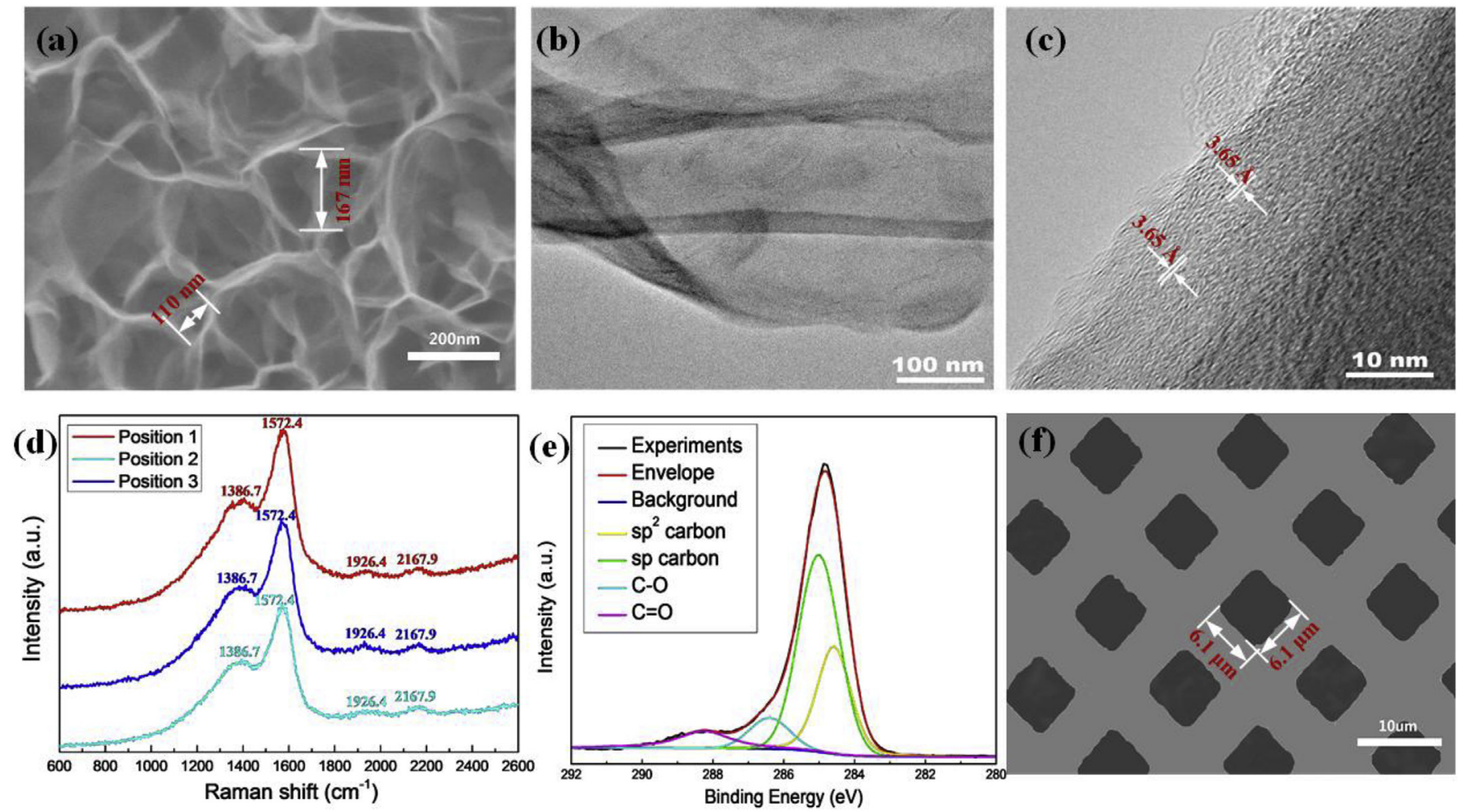

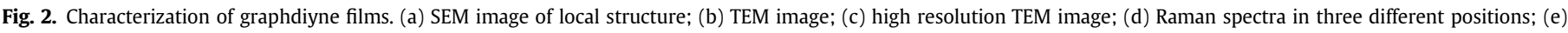
narrow scan for element C by XPS; (f) SEM image of copper network with rectangular wells. (A colour version of this figure can be viewed online.)

subtracted a Shirley background and fitted with a mixture function of Lorentzian and Gaussian. Fig. 2f shows the copper network with square wells with aside length of about $6.1 \mu \mathrm{m}$, where the graphdiyne film has been transferred to the copper network.

\subsection{Results of AFM experiments}

In AFM experiments, the transferred graphdiyne films with thickness varying from $10 \mathrm{~nm}$ to $30 \mathrm{~nm}$ on the copper substrates with numerous wells were moved in the Inverted Fluorescence
Microscope (IFM) instrument to investigate the effects of thickness on the elastic behavior. The typical measured relationship between the applied load and the deflection of the graphdiyne film is given in Fig. 3. With increasing indentation depth to about $650 \mathrm{~nm}$, the applied force on the graphdiyne film increased nonlinearly to about $7000 \mathrm{nN}$. The relationship between the applied force and the indentation depth could be fitted by a cubic polynomial function as described by Eq. (2-2), from which the elastic properties such as per-tension stress, 2D and 3D moduli could be obtained. Five graphdiyne films with different thickness were tested, and the 


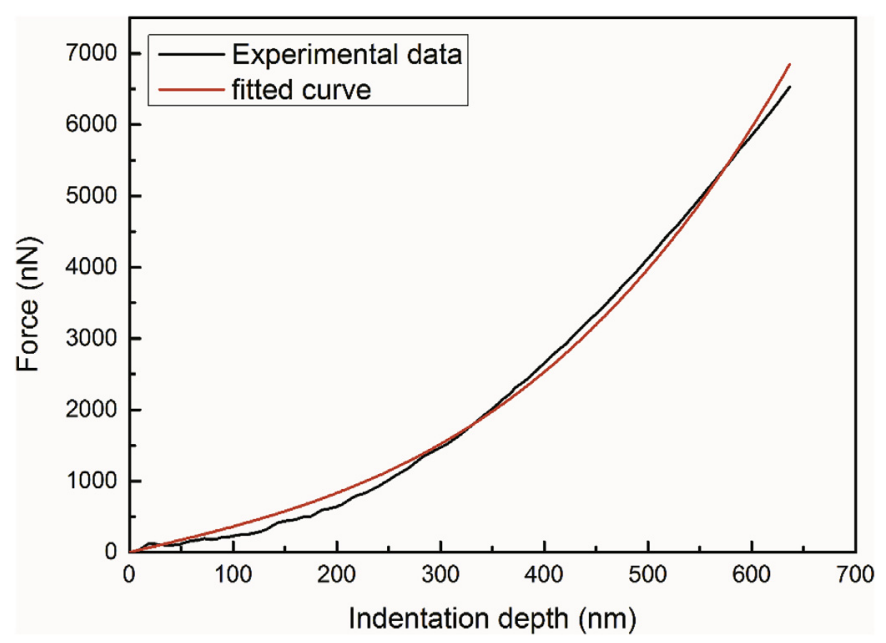

Fig. 3. Load versus indentation depth curve obtained from the experiment and fitted by Eq. (2-7). (A colour version of this figure can be viewed online.)

Table 1

Experimental results of different measured elastic modulus.

\begin{tabular}{llllll}
\hline Test No. & 1 & 2 & 3 & 4 & 5 \\
\hline 2D elastic modulus $(\mathrm{N} / \mathrm{m})$ & 53.1 & 74.5 & 82.9 & 88.3 & 99.8 \\
3D elastic modulus $(\mathrm{GPa})$ & 145.6 & 204.1 & 227.3 & 241.9 & 273.6 \\
\hline
\end{tabular}

results are given in Table 1. Due to the difference in thickness of the tested graphdiyne films, the corresponding 3D elastic modulus varied from $145.6 \mathrm{GPa}$ to $273.6 \mathrm{GPa}$, and the average 3D elastic modulus was $218.5 \mathrm{GPa}$.

\subsection{Results of $M D$ simulation}

The indenter located at the center of the graphdiyne film and moved perpendicularly to the surface of the graphdiyne film with a constant velocity. The atoms on the edge of the graphdiyne film were fixed. After loading a certain distance, some lattices of the graphdiyne film ultimately fractured and showed the unique failure mode.

\subsubsection{Bond length analysis}

The bond lengths of the model in the equilibrium after energy minimization were investigated to validate the MD simulation model. All the atoms were fully relaxed to the minimum energy state, and then the effective bond lengths were measured at the room temperature of $300 \mathrm{~K}$. A random selection of bond lengths of atoms except the fixed boundaries was averaged, resulting in a nearly constant value of $1.407 \AA$ for aromatic bonds, $1.340 \AA-1.395 \AA$ for single bonds, and $1.263 \AA$ for acetylene bonds, respectively. A brief summary of the calculated bond lengths is given in Table 2, in which the previous related results are also given as a comparison.

The acetylene bonds had been shown to change the character of aromatic benzene ring. The statistical results about the bond lengths showed that the $\mathrm{sp}^{2}$-sp jointed bond between the benzene ring and the acetylene linkage was elongated, indicating a weak conjugation effect between the benzene ring and the acetylene linkage. When compared to the typical lengths (about $1.40 \AA$ for aromatic bond and $1.54 \AA$ for single bond [44]), the equilibrium structure of graphdiyne showed that the aromatic bonds were elongated while the single bonds were shortened, which could be explained by the atomic interactions as the study by Cranford et al. [16] The measured triple bond lengths as given in Table 2 are a little larger than that in the previous published works, which could be ascribed to the difference in the adopted temperatures of systems. The higher temperature is, the more drastic thermal vibrations of atoms are, and correspondingly causing the larger bond lengths as obtained in the present simulation.

\subsubsection{Elastic modulus analysis}

The simulated force-deflection relationship of the graphdiyne films is depicted in Fig. $4 \mathrm{a}$, in which the radius of the indenter is $2 \mathrm{~nm}$ and the loading velocity is $0.2 \AA / p s$. The inset of Fig. 4 a gives the force-deflection curve of monolayer graphene [42] as a comparison. The fitted curves were based on Eq. (2-2). The maximum value of the indentation depth is $42.08 \AA$, corresponding to a critical force of $40.36 \mathrm{nN}$, which is consistent with the previous study [42] at the same simulation conditions. As shown in the inset of Fig. 4a, the fracture depth and the corresponding maximum force are $55.95 \AA$ and $655.08 \mathrm{nN}$, respectively, for the monolayer graphene. It could be seen that the maximum force of the monolayer graphene is much higher than the monolayer graphdiyne film whereas the indentation depth is almost the same, indicating that the strength of the graphdiyne is inferior but the ductility is superior to graphene. The difference of strengths between graphene and graphdiyne could be understood by the different number of bonds in structure. Each $\mathrm{C}$ atom is three-fold coordinated for graphene, whereas in graphdiyne the average coordination number of $C$ atom is 2.5 , leading to the relative smaller in-plane stiffness of graphdiyne. The ductility of graphdiyne should be ascribed to the introduction of the acetylene joint, providing a design method for the carbon family's 2D materials by introducing various types of acetylene. Fig. 4b shows the effect of loading velocity on the critical depth of the graphdiyne film. It could be seen that the influence of loading velocity is negligible in the present study range of velocity. Broad range of loading velocity conditions will be undertaken in near future to investigate the strain rate and inertial effects of the graphdiyne film.

The mechanical properties of the graphdiyne film were calculated according to Eqs. (2-2) to (2-7) and given in Table 3. The average value of elastic modulus, maximum stress of the graphdiyne film are $489.043 \mathrm{GPa}, 33.949 \mathrm{GPa}$, respectively. The elastic modulus in the present MD simulation agrees with the study of Refs. $[18,45,46]$ and the maximum stress is also consistent with Ref. [16].

It is to be noted that the elastic modulus is about $218.5 \mathrm{GPa}$ measured by AFM experiments, which is about half of its ideal

Table 2

Comparison of equilibrium bond lengths $(\AA)$.

\begin{tabular}{|c|c|c|c|c|}
\hline Works & $\mathrm{C}=\mathrm{C}$ & $\mathrm{C}-\mathrm{C}$ & $\mathrm{C} \equiv \mathrm{C}$ & Remarks \\
\hline graphdiyne & 1.407 & $1.340-1.395$ & 1.263 & MD, AIREBO \\
\hline Bai et al. [35] & 1.440 & $1.341-1.400$ & 1.239 & DFT, GGA-PBE, graphdiyne \\
\hline Pei [19] & 1.431 & $1.337-1.395$ & 1.231 & VASP, GGA-PBE, graphdiyne \\
\hline Yang and $\mathrm{Xu}[20]$ & 1.405 & 1.398 & 1.240 & MD, AIREBO, graphyne \\
\hline Haley [42] & 1.49 & 1.48 & 1.19 & MD, ReaxFF \\
\hline Cranford et al. [16,43] & $1.48-1.50$ & $1.46-1.48$ & $1.18-1.19$ & MD, ReaxFF; allotropes of graphyne \\
\hline
\end{tabular}



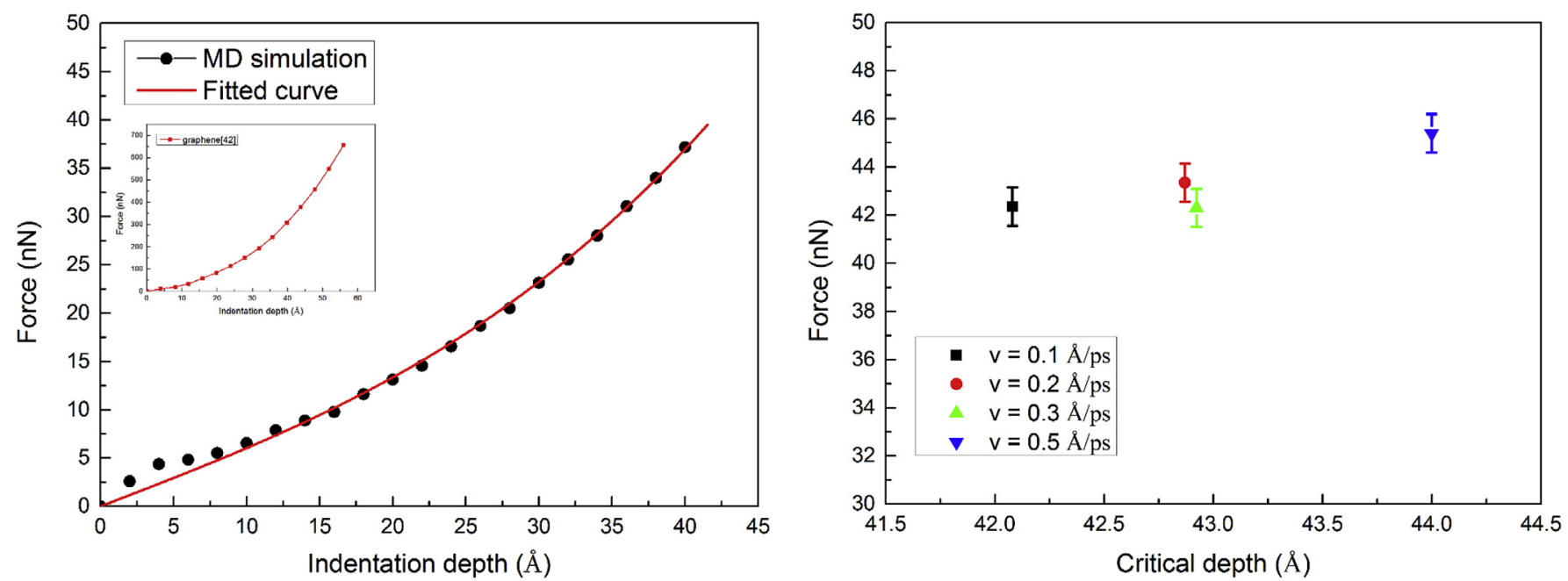

Fig. 4. a Load and indentation depth curves for the MD simulation results. b Critical depth and force for graphdiyne membranes.

Table 3

Mechanical properties of the graphdiyne film by nanoindentation MD simulation.

\begin{tabular}{llllll}
\hline Loading speed $(\AA / \mathrm{ps})$ & 0.1 & 0.2 & 0.3 & 0.5 & Average \\
\hline 2D elastic modulus $(\mathrm{N} / \mathrm{m})$ & 180.613 & 177.836 & 178.208 & 177.344 & 178.500 \\
3D elastic modulus $(\mathrm{GPa})$ & 494.829 & 487.224 & 488.241 & 485.876 & 489.043 \\
2D max stress (N/m) & 12.604 & 11.953 & 12.350 & 12.659 & 12.392 \\
3D max stress $(\mathrm{GPa})$ & 34.532 & 32.747 & 33.835 & 34.681 & 33.949 \\
\hline
\end{tabular}

value. The low measurement results should be ascribed to the effects of defects and thickness of the synthesized graphdiyne films. According to the study by Ahangari [18] and Ajori et al. [47], the elastic modulus of graphdiyne decreases significantly with the increase of vacancy atoms numbers. In addition, with the increase of layer numbers of the graphdiyne, the elastic modulus decreases dramatically $[48,49]$. It is worth noting that there are plenty of defects such as defects types, vacancy atoms numbers, defects locations and layer numbers. Systematically study on the effects of defects and layer numbers on the mechanical behavior of graphdiyne will be performed in near future.

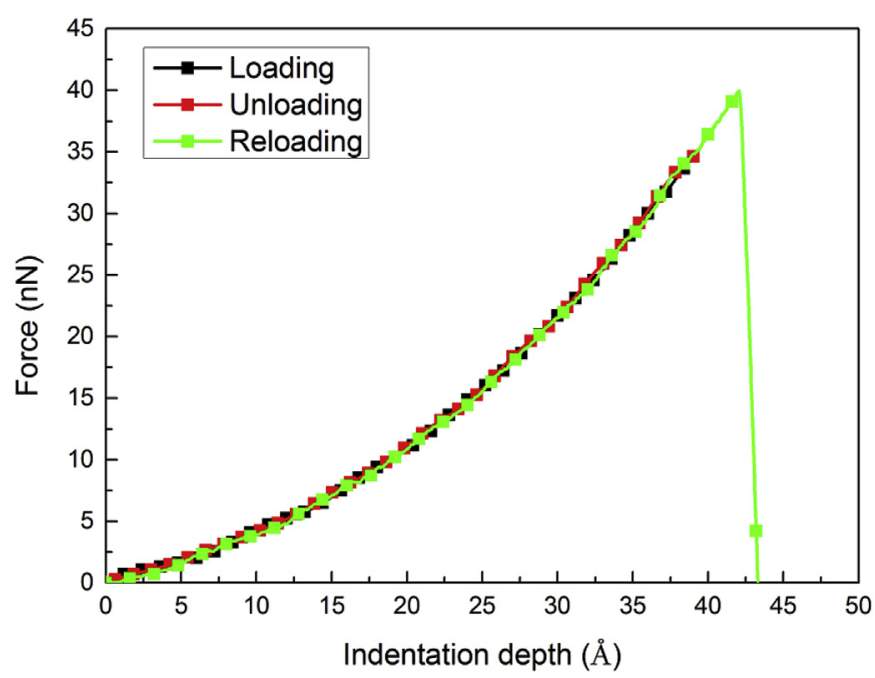

Fig. 5. Force-deflection curves of loading-unloading-reloading process. (A colour version of this figure can be viewed online.)

\subsubsection{Fracture behavior of the graphdiyne film}

The loading-deflection behavior of the graphdiyne film is given in Fig. 5, in which the loading-unloading-reloading process are also depicted. The loading and unloading curves of the graphdiyne film are almost identical, indicating the elastic behavior of the graphdiyne film. The reloading curve up to failure of the graphdiyne is also given in Fig. 5. It can be seen that with increasing the indentation depth to $42.08 \AA$, the load continuously increases to $40.36 \mathrm{nN}$ at the loading velocity of $0.2 \AA / \mathrm{ps}$. Then the abrupt drop of the reloading curve is observed with continuously loading, indicating the failure of graphdiyne film resulted from the broken of bonds between carbon atoms.

Fig. 6 illustrates the evolution of the lattice structures during fracture initiation of the graphdiyne film. The front and vertical views of the original atom structure are shown in Fig. 6a. When the indenter approaches the surface of the graphdiyne film, the graphdiyne film is convex due to the attraction between the indenter and the film if the distance is smaller than $3.4 \AA$. The graphdiyne film has a "snap-through" once the indenter contacts with the graphdiyne film. Similarly phenomenon has been observed in Refs. [32,50] With the increase of indentation depth, the force between indenter and the graphdiyne film changes from attractive interaction to repulsive interaction, and the $\mathrm{C}-\mathrm{C}$ bonds are getting longer as shown in Fig. $6 \mathrm{~b}$. With continuous indentation, the covalent bonds' lengths of the surrounding atoms of the graphdiyne film under the indenter begin to change. The acetylenic bond breaks firstly when the indentation depth exceeds the critical depth due to the lower binding energy of triple bond when compared to double bond and single bond, and the graphdiyne film expands largely as shown in Fig. $6 c-$ e. The first fractured bond of the triple bond is $\pi$ bond. Then some of the $\mathrm{C}=\mathrm{C}$ bonds break, and the fractured double bond is also $\pi$ bond. At last some $\mathrm{C}-\mathrm{C}$ bonds begin to break because the $\sigma$ bond of single bond is more stable than $\pi$ bond. Once the graphdiyne film is broken, the force between the indenter and the graphdiyne film suddenly drops to zero and the broken bonds are irreversible. After the indenter fully perforates the graphdiyne film, some broken atoms separate from the graphdiyne film and the irregular crack initiation is formed as shown in Fig. $6 \mathrm{~h}$.

It is interesting to note that the re-combination between the broken $\mathrm{C}-\mathrm{C}$ bonds and $\mathrm{C} \equiv \mathrm{C}$ bonds happens to sustain further deformation of the graphdiyne as shown in the highlighted black circle in Fig. 6f. In addition, the quadrangular structure as illustrated 


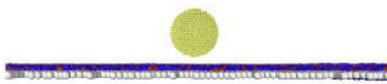

(a)
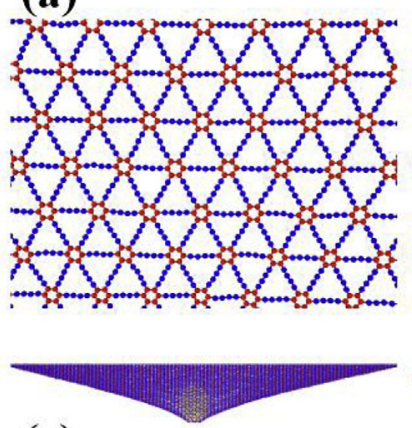

(e)

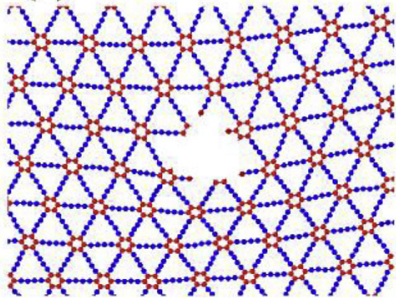

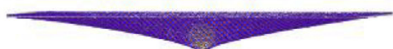

(b)

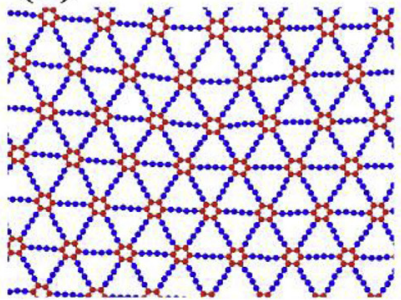

(f)

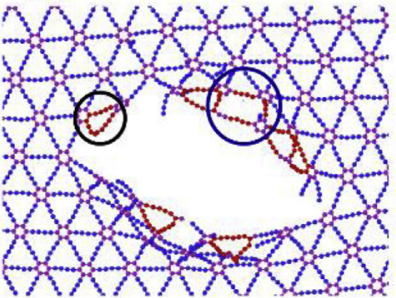

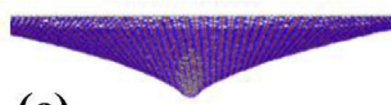

(c)

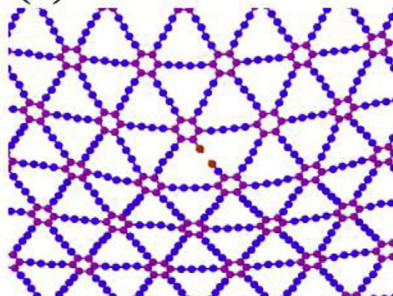

(g)

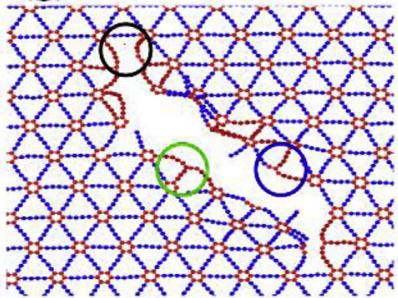

(d)
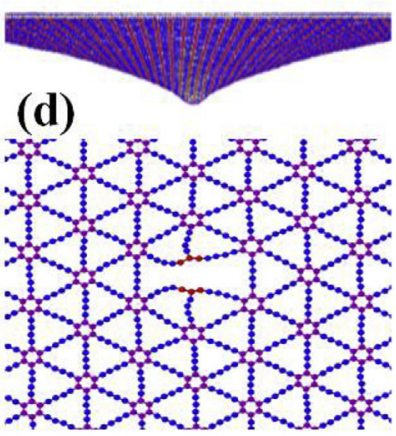

(h)

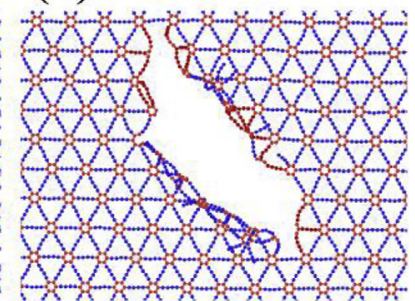

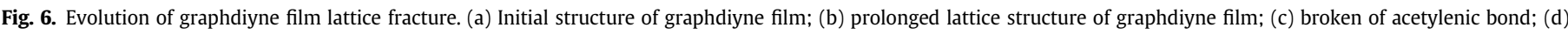

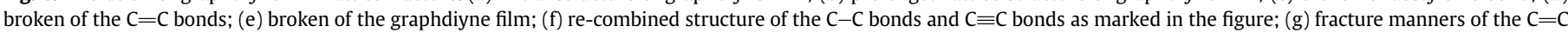
bonds as marked in the figure; (h) final broken behavior of graphdiyne film. (A colour version of this figure can be viewed online.)

in the blue circle as shown in Fig. $6 \mathrm{f}$ is observed. The broken $\mathrm{C}=\mathrm{C}$ bond is partially combined with the neighbor atoms to derive various unique shapes such as trilateral structure as shown in the highlighted circles in Fig. 6g. Be different from the symmetrical fracture behavior of graphene [32], the fracture of the graphdiyne film is asymmetrical as depicted in Fig. 6h, where a lathy crack is formed through bond breaking and recombining.

\subsubsection{Effects of loading parameters}

The effects of indenter radius and loading velocity on the mechanical properties of the graphdiyne film were also investigated

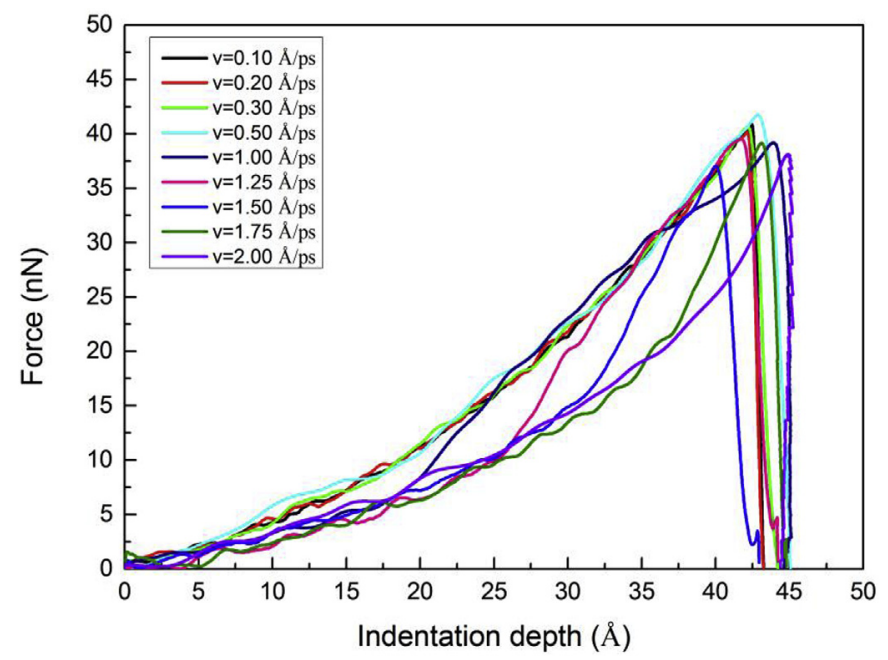

Fig. 7. Force-deflection behavior of graphdiyne film by changing indenter velocity. (A colour version of this figure can be viewed online.) through MD simulation. As shown in Fig. 7, it can be seen that when the indentation velocity is faster than $1 \AA / \mathrm{ps}$, the relationships between the force and the indentation depth are different from that obtained at indentation velocity slower than $1 \AA / p s$. With the increase of indentation velocity, the indentation depth increases slightly, whereas the critical loads are almost the same to each other. It is to be noted that there are some steep increases of slopes of the force-indentation depth curves at different indentation depth for each loading velocity, i.e. at $20.000 \AA$ for $1.00 \AA / \mathrm{ps}$, at $25.196 \AA$ for $1.25 \AA / \mathrm{ps}$, at $31.245 \AA$ for $1.50 \AA / \mathrm{ps}$, at $37.642 \AA$ for $1.75 \AA / \mathrm{ps}$, and at $43.872 \AA$ for $2.00 \AA / \mathrm{ps}$, respectively. This phenomenon could be understood by the vibration response of the graphdiyne film during impact. Once the indenter contacts with the graphdiyne film, the vibration wave is generated and propagates transversely. When it comes back to the contact region due to the reflection at the boundary, the force will increase abruptly as observed in Fig. 7.

The effects of indenter radius on the mechanical behavior of the graphdiyne film is given in Fig. 8a, where the radius of the indenter changes from 10 to $50 \AA$ and the same indentation velocity of $0.2 \AA /$ ps is employed. Larger indenter radius gives larger force and indentation depth. The basic mechanical properties, as shown in Fig. $8 \mathrm{~b}$, are calculated from these force versus indentation depth curves according to Eqs. (2-6) and (2-7) for $\mathrm{r} / \mathrm{a}<0.1$ (i.e. $\mathrm{r}<30 \AA$ ) and Eqs. (2-4) and (2-5) for r/a>0.1 (i.e. $r>30 \AA$ ). The calculated elastic modulus are slightly larger for $r>30 \AA$ than that for $r<30 \AA$ due to the effect of geometrical factor, $(r / a)^{1 / 4}$. However, there will be an obvious discontinuous point for $r / a=0.1$ according to Eqs. (2-4) to (2-7). Large error will be introduced into the analysis results for $r /$ $a \approx 0.1$ with the current analysis method as shown in Fig. $8 \mathrm{~b}$. Therefore, a revised equation as follows is developed to eliminate the discontinuous problem near $r / a=0.1$ while analyzing the mechanical properties of films, 

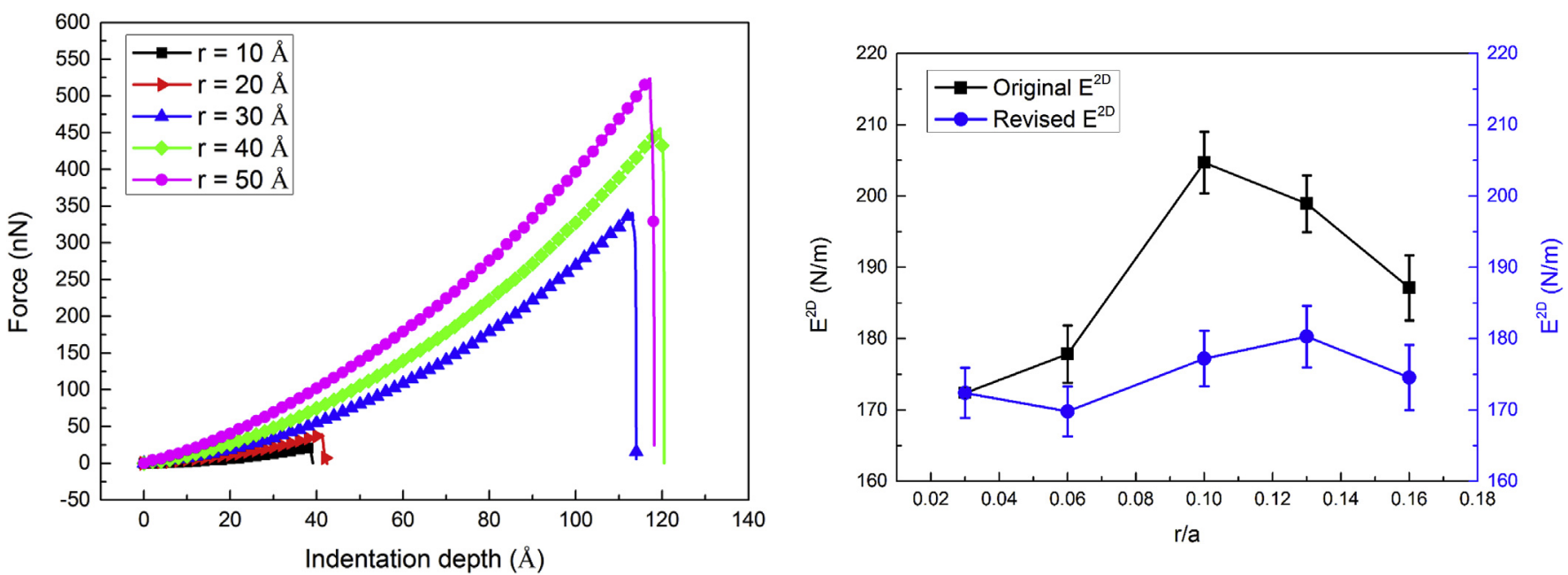

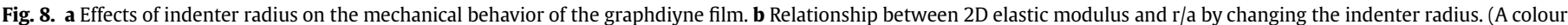
version of this figure can be viewed online.)

$F_{E^{2 D}}(\delta)=E^{2 D}\left(q^{3} a\right) \beta^{1 / 2}\left(\frac{r}{a}\right)^{\frac{1}{3}\left(\frac{r}{a}\right)^{1 / 4}}\left(\frac{\delta}{a}\right)^{3}$.

It is clearly shown that the error of $2 \mathrm{D}$ elastic modulus is significantly reduced according to Eq. (3-1) as depicted in Fig. 8b, demonstrating its effectivity for calculating the elastic properties of films in AFM experiments.

\section{Conclusion}

In the present study, the mechanical behavior of the graphdiyne film was studied by AFM experiments and MD simulation. The microstructure of the synthesized graphdiyne film was characterized, and the elastic modulus of the graphdiyne film was experimental measured to be about $218.5 \pm 72.9 \mathrm{GPa}$. The modulus of the grahpdiyne films showed dispersibility due to the initial defects during synthetic process. The simulated elastic modulus and the maximum stress of the perfect monolayer graphdiyne film were about $489.043 \mathrm{GPa}$ and $33.949 \mathrm{GPa}$, respectively. The failure behavior of the graphdiyne film was obtained in atomic level. It was observed that during the failure of the micro-structure, the acetylenic bonds broke firstly then the $\mathrm{C}=\mathrm{C}$ bonds and $\mathrm{C}-\mathrm{C}$ bonds broke subsequently. It was interesting to note that the broken $\mathrm{C}-\mathrm{C}$ bonds and $\mathrm{C} \equiv \mathrm{C}$ bonds recombined to sustain further deformation, and the broken $\mathrm{C}=\mathrm{C}$ bond partially combined with the near atoms to derive various unique micro-structures and failure pattern. Unlike graphene, the asymmetrical lathy crack was initiated in the graphdiyne film. The effects of indenter radius and loading velocity on the mechanical response of the graphdiyne film were also investigated. When the indentation velocity was faster than $1 \AA$ Aps, the slopes of force-deflection curves had steep increase points at certain indention depths for almost all the indentation velocity conditions simulated in the present study, which should be ascribed to the dynamic vibration response of the graphdiyne film. With increasing the radius of the indenter, the critical load and indentation depth also increased due to less stress concentration in the graphdiyne film. A revised formula was also developed for analyzing the mechanical properties of films in AFM experiments under various loading conditions.

\section{Acknowledgements}

The paper was supported by National Natural Science Foundation of China (Grant Nos. 11332011, 11672315, and 11772347), Science Challenge Project (Grant No. TZ2018001), and the Strategic Priority Research Program of the Chinese Academy of Sciences (Grant Nos. XDB22040302 and XDB22040303).

\section{Appendix A. Supplementary data}

Supplementary data to this article can be found online at https://doi.org/10.1016/j.carbon.2018.12.029.

\section{References}

[1] E. Mccann, V.I. Fal'Ko, Landau-level degeneracy and quantum Hall effect in a graphite bilayer, Phys. Rev. Lett. 96 (8) (2006), 086805.

[2] K.S. Novoselov, A.K. Geim, S.V. Morozov, D. Jiang, M.I. Katsnelson, I.V. Grigorieva, et al., Two-dimensional gas of massless Dirac fermions in graphene, Nature 438 (7065) (2005) 197.

[3] K.S. Novoselov, A.K. Geim, S.V. Morozov, D. Jiang, Y. Zhang, S.V. Dubonos, et al., Electric field effect in atomically thin carbon films, Science 306 (5696) (2004) 666-669.

[4] K.S. Novoselov, E. Mccann, S.V. Morozov, V.I. Falko, M.I. Katsnelson, U. Zeitler, et al., Unconventional quantum Hall effect and Berry's phase of 2 in bilayer graphene, Nat. Phys. 2 (3) (2006) 177-180.

[5] A.C. F, J.C. M, V. S, C. C, M. L, F. M, et al., Raman spectrum of graphene and graphene layers, Phys. Rev. Lett. 97 (18) (2006), 187401.

[6] R.H. Baughman, A.A. Zakhidov, W.A. de Heer, Carbon nanotubes-the route toward applications, Science 297 (5582) (2002) 787-792.

[7] A.H. Esbati, S. Irani, Probabilistic mechanical properties and reliability of carbon nanotubes, Arch. Civ. Mech. Eng. 18 (2018) 532-545.

[8] C. Lee, X. Wei, Q. Li, R. Carpick, J.W. Kysar, J. Hone, Elastic and frictional properties of graphene, Phys. Status Solidi 246 (11-12) (2009) 2562-2567.

[9] Y. Lin, W. Cai, X. Shao, Y. Lin, W. Cai, Fullerenes connected nanotubes: an approach to build multidimensional carbon nanocomposites, Chem. Phys. 331 (1) (2006) 85-91.

[10] T.H. Han, H. Kim, S.J. Kwon, T.W. Lee, Graphene-based flexible electronic devices, Mater. Sci. Eng. R Rep. 118 (2017) 1-43.

[11] M.S. Ribeiro, A.L. Pascoini, W.G. Knupp, I. Camps, Effects of surface functionalization on the electronic and structural properties of carbon nanotubes: a computational approach, Appl. Surf. Sci. 426 (2017) 781-787.

[12] R.H. Baughman, H. Eckhardt, M. Kertesz, Structure-property predictions for new planar forms of carbon: layered phases containing sp2 and sp atoms, J. Chem. Phys. 87 (11) (1987) 6687-6699.

[13] G. Li, Y. Li, H. Liu, Y. Guo, Y. Li, D. Zhu, Architecture of graphdiyne nanoscale films, Chem. Commun. 46 (19) (2010) 3256-3258.

[14] N. Narita, S. Nagai, S. Suzuki, K. Nakao, Optimized geometries and electronic structures of graphyne and its family, Phys. Rev. B 58 (16) (1998) 11009-11014.

[15] N. Narita, S. Nagai, S. Suzuki, K. Nakao, Electronic structure of three- 
dimensional graphyne, Phys. Rev. B 62 (16) (2000) 11146-11151.

[16] S.W. Cranford, D.B. Brommer, M.J. Buehler, Extended graphynes: simple scaling laws for stiffness, strength and fracture, Nanoscale 4 (24) (2012) 7797-7809.

[17] S.A. Hernandez, A.F. Fonseca, Anisotropic elastic modulus, high Poisson's ratio and negative thermal expansion of graphynes and graphdiynes, Diam. Relat. Mater. 77 (2017) 57-64.

[18] M.G. Ahangari, Effect of defect and temperature on the mechanical and electronic properties of graphdiyne: a theoretical study, Physica A 66 (2015) $140-147$.

[19] Y. Pei, Mechanical properties of graphdiyne sheet, Physica B 407 (22) (2012) 4436-4439.

[20] Y. Yang, X. Xu, Mechanical properties of graphyne and its family - a molecular dynamics investigation, Comput. Mater. Sci. 61 (2012) 83-88.

[21] G. Li, Y. Li, X. Qian, H. Liu, H. Lin, N. Chen, et al., Construction of tubular molecule aggregations of graphdiyne for highly efficient field emission, J. Phys. Chem. C 115 (6) (2011) 2611-2615.

[22] J. Kang, F. Wu, J. Li, Modulating the bandgaps of graphdiyne nanoribbons by transverse electric fields, J. Phys. Condens. Matter 24 (16) (2012), 165301.

[23] S.A. Elias, Global change impacts on the biosphere A2 - dellasala, dominick A, in: M.I. Goldstein (Ed.), Encyclopedia of the Anthropocene, Elsevier, Oxford, 2018, pp. 81-94.

[24] K. Srinivasu, S.K. Ghosh, Graphyne and graphdiyne: promising materials for nanoelectronics and energy storage applications, J. Phys. Chem. C 116 (9) (2012) 5951-5956.

[25] Y. Li, C. Guo, J. Li, W. Liao, Z. Li, J. Zhang, et al., Pyrolysis-induced synthesis of iron and nitrogen-containing carbon nanolayers modified graphdiyne nanostructure as a promising core-shell electrocatalyst for oxygen reduction reaction, Carbon 119 (2017) 201-210.

[26] Z.-Z. Lin, Graphdiyne as a promising substrate for stabilizing Pt nanoparticle catalyst, Carbon 86 (2015) 301-309.

[27] J. Li, T. Jiu, C. Duan, Y. Wang, H. Zhang, H. Jian, et al., Improved electron transport in MAPbI 3 perovskite solar cells based on dual doping graphdiyne, Nanomater. Energy 46 (2018) 331-337.

[28] Z. Meng, X. Zhang, Y. Zhang, H. Gao, Y. Wang, Q. Shi, et al., Graphdiyne as a high-efficiency membrane for separating oxygen from harmful gases: a firstprinciples study, ACS Appl. Mater. Interfaces 8 (41) (2016) 28166-28170.

[29] K.T. Wan, G. Shu, D.A. Dillard, A theoretical and numerical study of a thin clamped circular film under an external load in the presence of a tensile residual stress, Thin Solid Films 425 (1) (2003) 150-162.

[30] U. Komaragiri, M.R. Begley, J.G. Simmonds, The mechanical response of freestanding circular elastic films under point and pressure loads, J. Appl. Mech. 72 (2) (2005) 203-212.

[31] L. Xiang, S.Y. Ma, F. Wang, K. Zhang, Nanoindentation models and Young's modulus of few-layer graphene: a molecular dynamics simulation study, J. Phys. Appl. Phys. 48 (39) (2015), 395305.

[32] W. Wang, S. Li, J. Min, C. Yi, Y. Zhan, M. Li, Nanoindentation experiments for single-layer rectangular graphene films: a molecular dynamics study, Nanoscale Res. Lett. 9 (1) (2014) 41.

[33] N.M. Bhatia, W. Nachbar, Finite indentation of elastic-perfectly plastic membranes by a spherical indenter, AIAA J. 6 (6) (1968) 1050-1057.

[34] S. Plimpton, Fast Parallel Algorithms for Short-range Molecular Dynamics Academic Press Professional, Inc, 1995.

[35] H. Bai, Y. Zhu, W. Qiao, Y. Huang, Structures, stabilities and electronic properties of graphdiyne nanoribbons, RSC Adv. 1 (5) (2011) 768-775.

[36] S.J. Stuart, A.B. Tutein, J.A. Harrison, A reactive potential for hydrocarbons with intermolecular interactions, J. Chem. Phys. 112 (14) (2000) 6472-6486.

[37] H. Zhao, K. Min, N.R. Aluru, Size and chirality dependent elastic properties of graphene nanoribbons under uniaxial tension, Nano Lett. 9 (8) (2009) 3012-3015.

[38] D.W. Brenner, O.A. Shenderova, J. Harrison, S.J. Stuart, B. Ni, S.B. Sinnott, A second-generation reactive empirical bond order (REBO) potential energy expression for hydrocarbons, J. Phys. Condens. Matter 14 (4) (2002) 783-802.

[39] J. Li, X. Gao, B. Liu, O. Feng, X.B. Li, M.Y. Huang, et al., Graphdiyne: a metal-free material as hole transfer layer to fabricate quantum dot-sensitized photocathodes for hydrogen production, J. Am. Chem. Soc. 138 (2016) 3954-3957.

[40] J. Li, Z. Xie, Y. Xiong, Z. Li, O. Huang, S. Zhang et al., Architecture of betagraphdiyne-containing thin film using modified glaser-hay coupling reaction for enhanced photocatalytic property of TiO2, Adv. Mater. 29 (19) (2017), 1700421.

[41] R. Matsuoka, R. Sakamoto, K. Hoshiko, S. Sasaki, H. Masunaga, K. Nagashio, et al., Crystalline graphdiyne nanosheets produced at a gas/liquid or liquid/liquid interface, J. Am. Chem. Soc. 139 (8) (2017) 3145-3152.

[42] M.M. Haley, ChemInform abstract: synthesis and properties of annulenic subunits of graphyne and graphdiyne nanoarchitectures, Pure Appl. Chem. 80 (3) (2008) 519-532.

[43] S.W. Cranford, M.J. Buehler, Mechanical properties of graphyne, Carbon 49 (13) (2011) 4111-4121.

[44] J. Carper, The CRC Handbook of Chemistry and Physics, 1999.

[45] J.I. Klingemann, Mechanical properties of graphyne, Carbon 49 (13) (2011) $4111-4121$.

[46] R. Ansari, M. Mirnezhad, H. Rouhi, M. Faghihnasiri, Young's modulus and Poisson's ratio of monolayer graphene, J. Nanostru. 3 (3) (2013) 303-307.

[47] S. Ajori, R. Ansari, M. Mirnezhad, Mechanical properties of defective $\gamma$ graphyne using molecular dynamics simulations, Mater. Sci. Eng. 561 (2013) 34-39.

[48] Y. Zhang, C. Pan, Measurements of mechanical properties and number of layers of graphene from nano-indentation, Diam. Relat. Mater. 24 (2012) 1-5.

[49] I.W. Frank, D.M. Tanenbaum, A.M. van der Zande, P.L. McEuen, Mechanical properties of suspended graphene sheets, J. Vac. Sci. Technol. B: Microelect. Nanometer Struct. 25 (6) (2007) 2558.

[50] T.H. Fang, H.W. Tong, J.C. Yang, Y.J. Hsiao, Mechanical characterization of nanoindented graphene via molecular dynamics simulations, Nanoscale Res. Lett. 6 (1) (2011) 1-10. 